\title{
Metropolización y política urbana en la ciudad de México: en busca de un nuevo enfoque
}

\begin{abstract}
Alfonso X. Iracheta Cenecorta*
Este ensayo presenta una reflexión sobre la urgente necesidad de que se reconceptualice el carácter del proceso de la metropolización de la ciudad de México y se avance en su planificación estructurando una reforma urbana y creando un organismo metropolitano. Este último se encargaría de realizar todos aquellos servicios y obras de infraestructura que técnicamente requirieran hacerse en forma global para toda la zona metropolitana.
\end{abstract}

El tema de la excesiva concentración urbana en el área metropolitana de la ciudad de México (AMCM), ha tomado un nuevo impulso después de los dos terremotos de septiembre de 1985 que devastaron zonas importantes de la ciudad de México. Esto ocurrió en un contexto metropolitano en el que los problemas de suelo, vivienda, transporte y deterioro ambiental, han rebasado niveles críticos y donde el desempleo, el subempleo y otras patologías sociales han llegado a situaciones no tolerables.

Las declaraciones de funcionarios públicos en relación a este problema, si bien en muchos casos fueron de claro corte coyuntural, reflejaron la internalización de la crisis metropolitana en los aparatos del Estado, cuya respuesta se ha dado en torno al viejo dilema entre centralización y descentralización.

Es decir, se ha puesto nuevamente sobre la mesa de la discusión política la contradicción que ha caracterizado al crecimiento del AMCM en los últimos cuatro decenios. Por un lado, la ampliación de programas "desarrollistas" y "modernizadores" en los que se establecía que la concentración de las actividades de punta, principalmente en la capital del país, era un prerrequisito para el desarrollo nacional y que, por tanto, la expansión del área metropolitana se justificaba por el objetivo de alcanzar altos niveles de eficiencia económica similares a los exhibidos en los países industrializados de occidente. Por el otro lado, y como producto de las consecuencias negativas acumuladas históricamente, el convencimiento de que el AMCM se está convirtiendo progresivamente en una carga para el país y en un obstáculo para el desarrollo nacional, al absorber recursos del resto del territorio y, no obstante, ser incapaz de resolver las necesidades de su población y actividades económicas. Asimismo, por incurrir en costos sociales de urbanización cada vez mayores y por ser el centro

* Director de la Escuela de Planeación Urbana y Regional de la Universidad Autónoma del Estado de México. 
a través del cual se ejercen los controles del capitalismo internacional y del poder nacional sobre el resto de las regiones que conforman el país.

Las condiciones específicas que privan en el AMCM, hacen muy difícil aceptar que el enfoque de análisis y solución a su crisis se circunscriba preponderantemente a la cuestión de la descentralización, por las siguientes razones:

- La historia nos demuestra que las fuerzas determinantes del modelo de desarrollo nacional, en lo económico y en lo político, son opuestas a la descentralización.

-Al poner al frente de la discusión la dicotomía entre centralización y descentralización surge la tendencia a encubrir o soslayar dos cuestiones fundamentales: a) los límites estructurales y funcionales del modelo mexicano para desarrollar, gobernar y planificar el AMCM "hacia adentro"; b) el reconocimiento de la institucionalización e irreversibilidad del proceso de metropolización, dentro de las condiciones actuales, que hacen excesivamente difícil y costoso el éxito de programas de descentralización.

- La descentralización económica y administrativa como objetivo y como política estatal ha sido intentada en diversas ocasiones con muy pobres resultados.

Lo anterior induce a pensar que es conveniente intentar conceptualizar el fenómeno metropolitano de la ciudad de México desde una perspectiva que permita dar respuesta a múltiples interrogantes respecto del contenido, funciones y relaciones de este espacio hacia adentro y hacia afuera.

Si fuera posible conocer y explicar de manera específica cuáles son y de qué forma operan las fuerzas que han determinado la macrocefalia de la ciudad de México, el papel que han jugado en ella los diferentes agentes sociales que producen y reproducen el espacio, sus vinculaciones en el orden nacional e internacional y las perspectivas futuras que este espacio económico y físico presenta, estaríamos en mejores condiciones de proponer políticas que resuelvan o al menos reduzcan los problemas agudos que enfrenta.

Dentro de este contexto, las probables alternativas de las políticas de descentralización nos conducen a definir, cuando menos, dos enfoques propositivos: la descentralización de actividades del núcleo metropolitano hacia otras regiones del país; ${ }^{1} \mathrm{o}$ bien, el ordenamiento de dichas actividades dentro del espacio metropolitano urbanizado y urbanizable.

Si bien siempre será posible concebir un número de combinaciones entre ambas, el predominio de alguna de ellas deberá surgir de una per-

${ }^{1}$ El término descentralización implica, además de la localización de actividades de producción fuera del foco o región de referencia (desconcentración), el traslado de las actividades de decisión y administración pública y privada. 
sepción clara de la dirección que siguen las fuerzas que han determinado Il modelo de desarrollo nacional.

La pregunta es: $\Varangle$ hasta dónde es posible descentralizar actividades reevantes de la metrópolis cuando el fundamento mismo del sistema ecorómico y político es hacia la centralización?; o bien, ¿hasta dónde es po;ible ordenar las actividades metropolitanas en el espacio, con una jerspectiva de largo plazo, cuando esto puede implicar una restructuraión drástica del territorio metropolitano y su entorno?

No se cuenta con respuestas claras a estas interrogantes, probablemente porque no se cuenta con un conocimiento amplio y serio sobre el fenómeno metropolitano de la ciudad de México; y aunque los procesos socioespaciales y las acciones de planeación no se detienen por este hecho, es slaro que la metrópolis mexicana ha caído en un proceso de deterioro sumamente grave que nos obliga a retomar su análisis replanteando mucho de lo que hasta ahora se ha dicho y hecho.

\section{Desarrollo y metropolización: algunos conceptos}

Las primeras preguntas que surgen son: ¿qué es una metrópolis?, ¿podemos hablar de metrópolis en sentido genérico o más bien debemos orientar nuestro análisis a situaciones específicas?; lo que los estudiosos del fenómeno en países industrializados llaman metrópolis, ¿puede aplicarse a nuestro país?

Aun si encontramos respuestas adecuadas a estas preguntas, el problema de fondo no ha sido planteado. Es decir, cuando hablamos de áreas metropolitanas sólo nos referimos a un fenómeno físico-espacial que en virtud de su complejidad, de su gran tamaño, de su concentración poblacional y económica, de su expansión sobre diversas unidades políticoadministrativas, requiere de un tratamiento especial, o enfrentamos un fenómeno nuevo, cualitativamente distinto de lo que tradicionalmente llamamos urbano, y que rebasa la discusión meramente funcional para ubicarse en el nivel del proceso de desarrollo de una formación social concreta y las formas espaciales que adopta.

En el primer caso, la preocupación esencial es describir de manera "objetiva" un fenómeno y como resultado intentar definirlo y acotarlo. En otras palabras, al descubrir que una ciudad es muy grande, que ha rebasado sus límites político-administrativos, que destaca en relación con el resto del sistema de ciudades (a nivel regional o nacional), y que cumple un papel de polo económico, entre otras características, se le llama metrópolis, área metropolitana o región metropolitana.

En el segundo caso, la preocupación va más allá de la espacialidad del fenómeno y se concentra en aquellos determinantes de índole socio- 
económica y política que, vistos históricamente, producen y re producen algo que convencionalmente llamamos área metropolitana.

En este sentido la metrópolis, tal y como la conocemos, sería una forma básica de organización del capitalismo y, por tanto, su explicación estaría dada por las formas que éste ha adoptado ya sea en los llamados países capitalistas avanzados o en aquéllos que representan su contraparte histórica, los llamados países subdesarrollados o de menor desarrollo relativo (Castells, 1978: 32).

Si bien la metrópolis del mundo subdesarrollado presenta en su estructura física y funcional algunos de los rasgos observados en la metrópolis del mundo occidental industrializado (gran tamaño, polarización del espacio económico, alta concentración y crecimiento poblacional, rebasamiento de los límites administrativos originales, etc.), eso no qu iere decir que la explicación del fenómeno y las líneas de política gubernamental para la solución de sus problemas tengan que ser las mismas que han sido utilizadas en aquél.

El traslado de concepciones sobre el fenómeno metropolitano no toma generalmente en cuenta una cuestión básica "la de la aceleración del crecimiento urbano en las regiones subdesarrolladas con un ritmo superior al del despegue urbano en los países industrializados y ésta sin crecimiento económico concomitante" (Castells, 1978: 50).

Una interpretación de estos hechos nos conduce a estudiar la urbanización y por tanto la metropolización en los países "subdesarrollados" en relación con la investigación del "subdesarrollo mismo".

Por consiguiente, podríamos adoptar el término metrópolis, área metropolitana o región metropolitana como cuestión de método (en el sentido de Harvey, 1973), pero caracterizando al mismo tiempo los elementos específicos centrales que explican este fenómeno en México, a saber:

i) La metropolización de la ciudad de México no se da en un proceso de desarrollo como el ocurrido en los países industrializados, sino en un proceso de subdesarrollo que surge de la dependencia y de las formas concretas de acumulación a nivel nacional e incluso subnacional.

ii) La expansión de la ciudad de México hasta alcanzar la metropolización, es producto de la creciente migración campo-ciudad y de la concentración de las actividades modernas de nuestra economía subdesarrollada y no del progreso técnico y la industrialización.

iii) La terciarización de la metrópolis es un hecho que requiere ser cuidadosamente calificado. Mientras que en los países industriales es producto del desarrollo tecnológico y la modernización, en México es producto del subempleo y desempleo.

iv) En la gran metrópolis mexicana, coexisten todos los niveles de desarrollo conocidos, a diferencia de las metrópolis del mundo industrializado, donde se observa una cierta homogeneidad en la distribución de la riqueza y los beneficios de la urbanización. 
v) La ideología dominante ha hecho prevalecer el conflicto entre regiones atrasadas y regiones centrales (metropolitanas) por encima del conflicto entre clases poseedoras y clases desposeídas. Por consiguiente, las políticas enfocan la descentralización, la creación de polos que hagan contrapeso al área metropolitana y el mejoramiento en la distribución del ingreso entre regiones, subestimando las políticas de reducción de las desigualdades entre estratos sociales ubicados dentro del espacio metropolitano.

De ahí que la adopción del término metrópolis, área metropolitana o región metropolitana, para señalar un fenómeno de aparición relativamente reciente en nuestro país, nos obliga a aceptar de entrada que es éste un fenómeno novedoso, sin paralelo en la historia nacional.

Como tal, requiere de un tratamiento distinto, basado en una interpretación del desarrollo histórico concreto de la formación social donde aparece, del cual sea posible extraer los elementos de análisis para caracterizarlo conceptualmente.

Una primera cuestión es que el análisis del fenómeno metropolitano presenta tres niveles claramente diferenciados: el nacional, el regional y el metropolitano propiamente dicho. Esta distinción es solamente metodológica, ya que el estudio y la planificación del AMCM requiere necesariamente la consideración de los tres niveles.

El funcionamiento de la economía en el nivel nacional y sus vinculaciones con los centros internacionales de poder, son los elementos clave para comprender nuestro proceso de metropolización a escala nacional. Asimismo, es en el nivel nacional donde es posible situar las perspectivas de expansión física y demográfica de la metrópolis, dentro del contexto de las políticas generales de desarrollo del país.

El nivel regional corresponde al espacio económico-funcional directo del núcleo metropolitano, en el que se incluyen otros centros de población de diverso tamaño, así como espacios vacíos con usos no urbanos y cuyo límite es una línea virtual determinada por las relaciones de dependencia directa (adecuada accesibilidad e interacciones económicas intensas y constantes). Este nivel constituye el marco de análisis para sugerir las alternativas de expansión y ocupación territorial del núcleo metropolitano.

Finalmente, el nivel metropolitano propiamente dicho corresponde al espacio ocupado por la mancha urbana continua y las áreas inmediatas en proceso de urbanización o susceptibles de transformación a este uso.

En este nivel es posible definir con cierto detalle los factores que condicionan la organización y funcionamiento interno de la metrópolis y que son determinantes en su proceso de expansión y desarrollo futuro.

Una segunda cuestión es que los problemas tradicionales del AMCM requieren ser revisados, ya que ni todos son problemas en sí mismos, ni tienen la misma importancia relativa. De hecho, la definición de la pro- 
blemática metropolitana según el punto de vista que se elija, responde a posiciones particulares de interpretación del fenómeno y se sitúa, por lo tanto, en el campo de la ideología.

Así, por ejemplo, el problema mismo del subdesarrollo tiende a ser soslayado y reinterpretado por una conceptualización en la que se enfatiza que nuestro país está en proceso de desarrollo, y que se encuentra en transición hacia un desarrollo similar al de los países occidental es industrializados. Con ello se han justificado medidas de política que, sin afectar la actual situación de dependencia, procuran mejorar algunos índices cuantitativos de crecimiento, mismos que generalmente son acept ados dentro del sistema como índices de desarrollo. De la misma forma, se enfatiza el desequilibrio regional entre el área metropolitana y las zonas deprimidas del país por encima de los desequilibrios en la distribución del ingreso entre grupos sociales.

Otro ejemplo relevante lo constituye el excesivo crecimiento poblacional y la deficiente planeación territorial como "causas" de la problemática metropolitana. Las políticas de control natal, la expansión de los aparatos de planeación estatal y la producción de documentos de planeación (planes, programas y proyectos) adquieren entonces gran relevancia política.

Una tercera cuestión es que se pueden distinguir diversas características geoeconómicas peculiares al fenómeno metropolitano de la ciudad de México.

Destaca el que a este fenómeno lo caracterice su situación de núcleo o nodo en relación con su espacio geoeconómico regional o nacional. En este sentido, la metrópolis, como espacio urbano continuo, es sólo una parte del espacio geoeconómico mayor (región metropolitana), cuya delimitación estará en cada caso sujeta a la mayor o menor intensidad de flujos y relaciones entre el núcleo metropolitano y el sistema interurbano de su periferia inmediata.

En el caso de la ciudad de México, su área metropolitana contigua está delimitada por el Distrito Federal y 17 municipios del estado de México. Sin embargo, es probable que la región metropolitana que la contiene haya rebasado los límites geográficos de la cuenca de México hasta alcanzar algunas ciudades capitales de los estados vecinos.

Así, a medida que las facilidades de accesibilidad han ido creciendo y disminuye la "fricción del espacio", el ámbito de la región metropolitana se ha ampliado, incorporando espacios y subsistemas urbanos que antes presentaban una mayor autonomía relativa. Probablemente los casos más evidentes lo constituyen los valles de Toluca-Lerma y Cuernavaca.

Finalmente, con relación a la conveniencia de aplicar en nuestra metrópolis esquemas analíticos desarrollados en los países industrializados, saltan a la vista dos cuestiones: por un lado, la persistencia de una visión dominante respecto de los procesos de desarrollo y urbanización en los paí- 
es subdesarrollados, que se caracteriza por tener como premisa filosófica a preservación del statu-quo y, por otro lado, la evidencia histórica de jue nuestro país ha seguido un patrón de desarrollo cuyas características :entrales son totalmente diferentes de las de los países capitalistas desarollados.

Como consecuencia de lo anterior, el traslado aeritico y directo de teoias, métodos y técnicas de análisis y planeación metropolitana de una ealidad (el desarrollo capitalista) a otra (el subdesarrollo capitalista), reiulta muy cuestionable.

\section{Problemas metropolitanos relevantes}

Jna de las repercusiones más evidentes de la profunda crisis en que se mcuentra México es la reducción de los niveles de vida de la mayoría de la población.

En el medio urbano, en general, y en las grandes metrópolis, en particular, esta situación se hace más clara al observarse:

- Una creciente concentración de la renta en los tramos más altos de la pirámide social, sumiendo en la miseria absoluta a aquellos grupos que ya de por sí eran los menos favorecidos. Al mismo tiempo, se reducen sensiblemente los niveles de vida de los grupos sociales medios (asalariados, profesionales, burócratas) que, de clase media afluente, transitan progresivamente hacia la proletarización.

-Un sensible incremento en el desempleo urbano, con la consecuente movilización de fuerza de trabajo al llamado "sector informal" de la economía urbana.

- La mayor especialización del circuito económico moderno, acorde con la creciente concentración del ingreso, mediante el traslado de los países centrales de tecnologías y formas de organización más sofisticadas.

-Una reducción en las inversiones estatales orientadas a la producción de medios de consumo colectivo (escuelas, hospitales, transporte, parques, etc.), como consecuencia de la imposición por parte de los organismos internacionales de crédito, de modelos económicos recesivos.

-Una disminución en la ya de por sí escasa prioridad que el Estado da a la problemática metropolitana frente a los problemas de deuda externa, inflación, balanza de pagos, etcétera.

Esta profundización de la permanente crisis del subdesarrollo de nuestro país, está teniendo múltiples consecuencias en la configuración espacial metropolitana, especialmente de la ciudad de México.

Nuevos problemas aparecen y los que ya existían se agudizan ante una aparente pérdida de capacidad por parte del Estado y de las clases dominantes para mantener el precario equilibrio de las contradicciones espaciales metropolitanas. 
Esta situación nos lleva a repensar los problemas metropolitanos y a redefinir sus prioridades.

Ahora más que nunca, el problema económico es el que se sitúa al frente de la crisis urbano-metropolitana. Por esto entendemos no sólo la agudización del desempleo abierto y el subempleo y la creciente concentración de la riqueza, sino la incapacidad del sistema para mantener un cierto equilibrio en el proceso de producción, distribución y consumo metropolitano.

En otras palabras, nuestra metrópolis ha dejado de ser la gran productora de empleo para ofrecer apenas un espacio para el subemp leo "informal" con niveles muy precarios de ingreso. El desarrollo económico metropolitano es cada vez más especializado y más volcado al circuito moderno de la economía, que se caracteriza por sus altos niveles de organización y desarrollo tecnológico, y por ofrecer bienes y servicios para los grupos de mayor ingreso de la sociedad. La brecha entre éste y el circuito más tradicional de la economía metropolitana tiende a ensancharse y la dominación del primero sobre el segundo a perpetuarse.

El problema de la distribución territorial de la población y las actividades económicas está íntimamente ligado al anterior. Las activida des más rentables y por ende las más atractivas para el capital, se localizan en los mismos puntos privilegiados del espacio, donde las ventajas de localización les garantizan una ganancia mayor. Las actividades inferiores o tradicionales dependen de esta lógica de distribución espacial basada en criterios de ganancia económica, al quedar limitadas a absorber los espacios que no ocupa la economía dominante, aquéllos donde se ubica la población de menor ingreso y que tienen las mayores desventajas para la producción y el consumo.

El problema del consumo colectivo se deriva de los anteriores. Los bienes de consumo colectivo generalmente no son rentables para el capital privado y así su producción queda en las manos del Estado. La lógica seguida por éste, sin embargo, hace que se produzcan bienes y servicios de consumo colectivo en la medida en que son "útiles" al proceso productivo, es decir al capital, y no en la medida en que sean parte de un proceso de reducción de las desigualdades sociales en la metrópolis.

Se producen así grandes obras viales o complejos habitacionales para los grupos de ingreso medio y alto, mientras que las condiciones sanitarias de los barrios más pobres permanecen sin solución.

De ahí que el problema del consumo colectivo metropolitano esté alcanzando un nivel en el cual las soluciones tradicionales son definitivamente insuficientes. Ya no basta con establecer programas de suelo, vivienda y de servicios públicos y sociales para colonias populares. Cuantitativa y cualitativamente, estos problemas han rebasado con mucho la acción del Estado y, bajo los esquemas actuales de distribución de re- 
ursos públicos y de determinación de las políticas metropolitanas, son :laramente insolubles.

Otros problemas metropolitanos más ligados a la producción que al :onsumo colectivo, como es el caso del transporte, presentan mayores poibilidades de solución en la medida en que las actividades económicas meropolitanas dependen de su adecuado funcionamiento para su desarrollo.

Del mismo modo, al problema de la coordinación institucional entre Jrganismos y niveles de gobierno que actúan en el ámbito metropolitano zo se le otorga, en las prioridades estatales, la gran relevancia que reviste m la medida que dicha coordinación es fundamental dentro del proceso de acumulación global. En este sentido, destaca observar cómo la organización político-administrativa no ha cambiado en el AMCM, a pesar de que la expansión de la mancha urbana ha rebasado en mucho los límites del Distrito Federal hasta cubrir un gran número de municipios del estado de México.

Finalmente, resulta sorprendente que la provisión y administración de los servicios públicos y el equipamiento, así como las tasas de planeación, no consideren una escala metropolitana-regional, no obstante las claras demandas surgidas de la agudización de los propios problemas y carencias en ese nivel de organización espacial.

Por lo anterior, es posible establecer la hipótesis de que la definición de los problemas metropolitanos, tal como se propone desde las estructuras dominantes, tiene un carácter ideológico. En este sentido, dichos problemas y las acciones consecuentes para su solución no corresponden a las necesidades objetivas de la mayoría de la población, sino que son priorizadas fundamentalmente desde la perspectiva de las necesidades de acumulación del capital.

En otras palabras, aquellos problemas que se encuentran en la base del proceso de reproducción de la fuerza de trabajo, como es el caso del agua, el drenaje, la vivienda, la salud y la recreación, adquieren prioridad sólo cuando el proceso de acumulación se ve amenazado por la no correspondencia entre el crecimiento de la economía y la base trabajadora que lo sostiene.

Una economía metropolitana en proceso de modernización y orienta$\mathrm{da}$ al consumo masivo requiere de una base poblacional que garantice la expansión de su mercado. Los niveles de vida de esa población deben estar acordes con el nivel de desarrollo de dicha economía y si, como ya se mencionó, la fuerza motriz de ésta se encuentra en el circuito moderno y no en el tradicional, resulta lógico que sean los grupos sociales de mediano y alto ingreso los que se beneficien por las acciones de planificación metropolitana.

Existen evidentemente otras circunstancias que operan en la reorientación de las acciones de planificación hacia los grupos de menor ingreso. Éstas, sin embargo, dependen fundamentalmente de los niveles de or- 
ganización y politización de los trabajadores y pobladores, y de su capacidad concreta de movilización para reivindicar sus necesid ades más importantes.

\section{Carácter parcial de la actual planificación metropolitana}

Ante la pauperización masiva de buena parte de la población m etropolitana, la destrucción ecológica del espacio, la actuación irrestricta en el mercado de suelo y edificios, y la consecuente segregación socioespacial de las clases de menor ingreso, el Estado continúa aplicando programas puntuales con poca coordinación y administrando el espacio metropolitano con criterios parciales en muchos casos basados en la rentabilidad de las inversiones, lo que no corresponde a la problemática social metropolitana.

Pensamos que detrás de esto existen cuatro cuestiones fundamentales. Por un lado, todavía no hay en nuestro país una necesidad imprescindible por hacer de la planeación metropolitana un instrumento de socialización y corrección de las contradicciones propias del sistema. Es decir, no se ha llegado al momento histórico en que la planeación metropolitana permite expandir las fronteras de la acumulación y al mismo tiempo expandir los niveles de reproducción de la fuerza de trabajo reduciendo algunas de las desigualdades sociales.

Esto no quiere decir que no exista una planificación del desarrollo metropolitano, sino que es una planificación fundamentalmente orientada a institucionalizar la preeminencia del mercado privado de suelo y edificios. La planificación más orientada a los medios de consumo colectivo, capaz de definir y acotar el campo de la especulación inmobiliaria y al mismo tiempo basada en el concepto de servicio público y por tanto volcada a reducir algunas de las más agudas contradicciones socioespaciales, todavía no se materializa en el AMCM.

Una segunda cuestión, íntimamente ligada a la anterior, es la dominación de una tecnología para planificar el desarrollo urbano que no corresponde a las condiciones de la urbanización en nuestro país. Desde la definición del concepto de metrópolis, área metropolitana o región metropolitana, hasta en la selección y priorización de sus problemas, se observa, como ya se mencionó, la persistencia de posiciones descriptivas y aeriticas que ayudan poco a comprender el fenómeno.

No se trata de desechar lo que hasta ahora se ha alcanzado en este campo, sino de romper con una línea de pensamiento basada en gran medida en experiencias ajenas, y de crear una propia. Sólo bajo este hilo conductor será posible aprovechar parte de lo que las escuelas dominantes han desarrollado hasta ahora.

La tercera cuestión, que a nuestro juicio juega el papel más importante en la redefinición del desarrollo metropolitano, es la gestión populär. 
Vo existen dentro de los esquemas estatales de planificación metropolita1a espacios suficientes y adecuados para que la comunidad organizada jarticipe de la gestión del espacio que habita. La verticalidad y el autoriarismo son todavía las fuentes más importantes de decisión en las accioles relativas al desarrollo socioespacial. Más aún, en la ciudad de México os movimientos populares de base territorial que reivindican el derecho 1 la ciudad son incluso reprimidos, no obstante que son los interlocutores nás válidos para iniciar un proceso de transformación de la estructura iocial.

Una última cuestión se refiere a la administración y gobierno de la siudad de México. Nos encontramos apenas en una etapa de planteamiento iel problema metropolitano, sin que existan todavía conceptualizaciones , formas concretas para el tratamiento de la administración de la ciudad. Je hecho, sólo en los últimos años se han intentado formular ciertas políicas comunes y coordinar esfuerzos en materia de planeación y realiza:ión de algunas obras públicas entre el Distrito Federal y el estado de Mécico. No obstante, siguen predominando los enfoques particulares de cada mtidad, persistiendo, por lo tanto, una fragmentación artificial del espaio metropolitano.

Probablemente la formación de múltiples organismos y empresas esatales responsables de la provisión de servicios públicos y equipamiento netropolitano es la característica más relevante de las actuales tendenzias de la administración pública para enfrentar de manera organizada los Jroblemas y carencias de la población. Destaca, asimismo, la búsqueda jor hacer de estas empresas y organismos entidades cada vez más autofiuanciables, al prevalecer en las políticas financieras gubernamentales los riterios derivados de los programas impuestos por los organismos interracionales de crédito y financiamiento.

Si bien la eficiencia económica es importante, no es el único factor jue debe valorarse. La justicia social, bajo un esquema de interpretación eórica menos funcional y más estructural del proceso metropolitano, llevaría seguramente a cuestionar la operación de estas empresas estatales jara proveer servicios metropolitanos bajo la óptica exclusiva de la rentajilidad económica. Una de las consecuencias de estos esquemas adminisrativos es que sólo tienen derecho a la ciudad aquéllos que pueden pagar zor él. Los que están al final de la fila por su mínimo poder de compra, implemente están al margen de obtener los satisfactores que dichas emjresas producen.

l. Reconceptualización del fenómeno metropolitano: racia una reforma urbana

Inte la heterogeneidad y contradicciones internas del Estado, el capital I la sociedad civil, se requiere identificar, analizar y aprovechar los res- 
quicios existentes que permitan llevar a la práctica algunas tra nsformaciones en el proceso de desarrollo metropolitano.

En concreto, existen cuando menos tres tipos de acciones en las cuales es posible enmarcar las transformaciones más viables: en la reconceptualización teórica del fenómeno metropolitano; en la intervención del Estado, y en la participación y autogestión comunitaria de las decisiones que afectan el espacio habitado.

Estas tareas estarían estrechamente ligadas a un esquema de organización de la administración pública que tuviera una escala regionalmetropolitana, rebasando la fragmentación actual en múltiples unidades políticas administrativas, y en organismos y empresas en las que participan los distintos niveles de gobierno. En otras palabras, se requiere iniciar un proceso de transformación profunda de los conceptos políticos y organizacionales que hasta ahora han comandado el desarrollo metropolitano.

A este respecto, la pregunta más importante es: ¿cómo podríamos lograr, desde la estructura estatal, una más adecuada conceptualización del fenómeno metropolitano, una mayor y más decidida intervención del Estado en su desarrollo, y una real y efectiva gestión popular de estos espacios? La respuesta a estas cuestiones depende del grado de avance en las relaciones sociopolíticas en el país y en el AMCM en particular. Asimismo, depende de las estructuras organizativas y administrativas existentes y de su capacidad de transformación.

\subsection{Cambios en la conceptualización del problema metropolitano}

La reconceptualización de la planeación metropolitana comprende dos medidas. La primera es reconocer los procesos de desarrollo del AMCM en el contexto nacional y regional, estableciendo políticas adecuadas para ordenarlos más coherentemente. La segunda es establecer algunas acciones importantes tendientes a afectar los procesos de producción y reproducción, control y apropiación del suelo metropolitano.

La primera medida parte del supuesto de que las políticas de desarrollo metropolitano a nivel regional y nacional requieren enfocarse de manera distinta, rompiendo con el esquema reivindicativo del resto de las regiones subnacionales frente a la gran metrópolis nacional. La gran metrópolis, enmarcada en su espacio regional (región metropolitana), deberá perseguir objetivos que, tomando como base las metas nacionales de equilibrio espacial y social, se oriente a un crecimiento controlado y acotado. Como consecuencia, los procesos "naturales" de suburbanización de la metrópolis hacia su espacio regional adquieren gran importancia frente a la creación de nuevos "polos de desarrollo" hacia donde se busca descentralizar actividades metropolitanas. 
La segunda medida se basa en el supuesto de que, al interior del essacio metropolitano, el control sobre el suelo urbano y urbanizable adjuiere un carácter estratégico para poder efectivamente conducir y conrolar la expansión metropolitana.

\subsubsection{Regionalización de las políticas metropolitanas}

Ja adopción de políticas metropolitanas de carácter regional se derivará lel hecho objetivo de que el AMCM seguirá creciendo y expandiéndose a $\mathrm{m}$ ritmo elevado. Dicha expansión está adquiriendo un carácter de sumrbanización a escala regional a partir del núcleo metropolitano. El "salto sspacial" que están dando algunas actividades, provoca ciertos niveles le especialización (industria en Toluca-Lerma y turismo e industria en Juernavaca). La inducción de mejoras en la accesibilidad hacia Toluca-erma y la probable introducción de medios de transporte más rápidos l eficientes, reduciendo la fricción del espacio, traerá como consecuenia la aceleración de este proceso. ${ }^{2}$

Esta dinámica metropolitana, provocada por las necesidades de exjansión espacial de la base productiva y por la acción estatal, requiere ;er internalizada por las políticas estatales desde una perspectiva de plaseación regional metropolitana, en lugar de la actual, cuyo discurso es a descentralización no obstante que en las acciones se promueve la suJurbanización.

Es decir, con un discurso cuya contraparte práctica debería ser la creasión de polos lejanos que se opusieran al desarrollo metropolitano, y que jor lo tanto se evitara la expansión hacia los centros intermedios dentro iel ámbito regional, las políticas concretas que los gobiernos federal y esatal realizan van precisamente en la dirección opuesta, sin el reconociniento explícito de ello y sin la elaboración de los fundamentos teóriconetodológicos y técnicos que avalen dichas acciones.

De ahí que se requiera replantear las bases del planeamiento metrojolitano reconociendo, en primer lugar, que la suburbanización de su nú:leo y la planeación de este proceso a partir de un enfoque de región meropolitana puede ser una vía para la expansión controlada y más ordenada iel AMCM que, de cualquier forma, seguirá creciendo hasta alcanzar proJablemente entre 26 y 30 millones de habitantes al final del siglo.

En segundo lugar, se requiere replantear las bases para la desconcentración y descentralización, considerando para ello que la especialización de regiones (incluida la región metropolitana), más que la promoción de

\footnotetext{
2 La creación del aeropuerto internacional en Toluca, la ampliación de las carreteras que a unen con la ciudad de México y el probable desarrollo de un tren rápido entre Toluca y Vaucalpan son elementos aceleradores de esta suburbanización discontinua. El tren electrifisado a Querétaro probablemente tendrá efectos similares.
} 
todo tipo de actividades en cada región, se ajusta a las metas nacionales de equilibrio espacial.

Con ello sería posible situar en su justo nivel la dicotomía entre centralización y descentralización y, por ende, entre metrópolis y regiones subnacionales, dando prioridad a los problemas más relevantes que se sitúan en la necesidad de generar una restructuración del espacio económico y las relaciones sociales en busca de una distribución más equitativa de la riqueza entre estratos de la sociedad.

\subsubsection{Políticas de control sobre el suelo metropolitano ${ }^{3}$}

Estas medidas, como ya se mencionó, pretenden resolver algunos de los problemas más importantes relacionados con el suelo metropolitano y rebasan los actuales esquemas de acción, tanto estratégicos como operativos, pero son posibles de realizar dentro del modelo sociopolítico del país.

Sin pretender ser exhaustivos, se abordan aquellas cuestiones que, a la luz de los problemas del AMCM, se consideran como limitaciones o barreras para el tratamiento de otros problemas y necesidades correlacionadas. Tal es el caso de nuevas formas de regularización de la tenencia de la tierra, de diferentes formas de propiedad social del suelo metropolitano, de apertura de nuevas opciones de organización para el aprovechamiento del suelo, y finalmente, de la necesidad de revisar el marco jurídico y administrativo para el tratamiento del suelo metropolitano.

La manifestación superior de esta reconceptualización sería sin duda una reforma urbana. Por analogía con la reforma agraria, gestada en las luchas populares por el derecho a disponer de tierra y en contra del latifundio y la explotación, en el contexto de la metrópolis surge como necesidad ineludible obtener un pedazo de tierra urbana para vivir y para contribuir al desarrollo de las actividades productivas urbanas, como la industria, el comercio y los servicios.

A modo de ejemplo, algunas medidas que podrían ir dando forma a un proyecto de reforma urbana, serían las que a continuación se señalan:

i) El logro de una planificación real de los usos del suelo, tendiente a una reforma urbana integral en el futuro, debe partir de una nueva conceptualización estatal y social de la propiedad. La masificación de los problemas de urbanización popular y las limitaciones estructurales para allegarse suelo urbano, imponen que los esfuerzos estatales de dotación de tierra se dirijan prioritariamente a la satisfacción de las necesidades de

\footnotetext{
${ }^{3}$ Un análisis profundo de los problemas del suelo metropolitano y un argumento más elaborado sobre la necesidad de incidir en el mercado privado del suelo y edificios como precondición para un desarrollo más ordenado del AMCM, aparece en un libro que publicamos recientemente (Iracheta, 1984), cuyos datos fueron actualizados en otro trabajo (Iracheta, 1987).
} 
as clases populares. Es decir, se propone una clara división entre tierra ırbana y urbanizable para habitación, servicios y equipamiento, para los zrupos de menor ingreso y la orientada a otros usos. La primera estaría jajo estricto control estatal sujeto a congelación de su valor y posesión nmediata y, por lo tanto, fuera del mercado de tierra y edificios, y aunme la segunda estaría sujeta a los lineamientos estatales de planeación, 31 mercado privado inmobiliario podría operar en sus procesos de disribución y consumo.

ii) Para hacer efectivo lo anterior, se hace necesario diseñar nuevas ormas de apropiación del suelo urbano, así como que el Estado garantice a los sectores sociales beneficiados la posesión de éstas. Se ha hecho evidente que no es suficiente que una familia de bajo ingreso acceda a un iote urbano, por la vía de la compra legal o la regularización de la tenenzia. Se requiere garantizar, asimismo, que dichos sectores no sean expul¡ados por la vía del mercado a zonas cada vez más periféricas e insalubres.

iii) Los mecanismos de regularización de la tenencia de la tierra urJana requieren ser transformados.

iv) Resulta necesario adecuar los sistemas de planeación urbana que speran actualmente en el Distrito Federal y en el estado de México, integrándolos en su sistema de planeación metropolitana. Para ello resulta fundamental un replanteamiento del concepto de desarrollo metropolitano sobre bases más reales surgidas de los procesos socioeconómicos y espaciales que caracterizan al AMCM, así como abrir a la discusión pública la democratización del gobierno del Distrito Federal y la reorganización de los sistemas de administración metropolitana. Paralelamente, las transformaciones a los sistemas de planeación metropolitana deberían definir criterios adecuados y flexibles que permitan regular el proceso de formación de asentamientos populares, ya que más de la mitad de la población metropolitana habita en ese tipo de asentamientos.

v) Se requiere revisar las diversas leyes, reglamentos, bandos, etc., que regulan el desarrollo urbano, buscando la promulgación de una sola ley que surja de un claro concepto de planeación del desarrollo metropolitano y no de conceptos parciales sobre recaudación, incentivos o controles de situaciones específicas.

Esto implica, asimismo, replantearse a fondo el sistema de administración, fiscalización y financiamiento del AMCM con el fin, por un lado, de unificar los distintos esfuerzos en un todo coherente y, por otro, de revisar los criterios generales políticos y sociales que rigen estas acciones estatales, a fin de buscar un mayor equilibrio en la distribución de las cargas y beneficios del desarrollo metropolitano.

Indudablemente, se parte de la base de que todas estas medidas deberían encuadrarse en un plan de desarrollo metropolitano, ya que éste constituye, por definición, la forma de intervención estatal más elaborada y coherente. Sin la concepción estratégica de un plan, con determinacio- 
nes en cuanto a usos, destinos y reservas, así como lineamientos programáticos, no es posible lograr la concurrencia entre niveles de gobierno ni la concertación de acciones con los sectores privado y social.

\subsection{Participación popular}

Todas las medidas y acciones para el desarrollo metropolitano, requieren de aceptación política, y su factibilidad está asociada con la fuerza relativa de los diversos grupos sociales que intervienen como agentes relevantes del proceso socioespacial.

Si existen objetivos contradictorios, una crónica crisis urbana y la voluntad explícita de democratizar la acción gubernamental, podemos decir que hemos llegado al punto en que resulta insoslayable una auténtica participación popular.

Participar en la planeación y desarrollo de la metrópolis implica disponer de información, interiorizarse de planes y proyectos, generar alternativas y comprometerse con las soluciones adoptadas.

En relación con esto, valgan algunas consideraciones generales. Debe descartarse la idea de que promover la participación popular es fabricar un auditorio silencioso para escuchar y convalidar decisiones ya tomadas o, menos aún, involucrar a la población como mano de obra gratuita para la urbanización. Un verdadero proceso participativo en la gestión metropolitana empieza por la base misma del problema y requiere de una estructura con instancias y niveles que opere en forma permanente. De no ser así, los grandes eventos organizados en torno a temas imprecisos y globales sólo generan interminables intervenciones que reiteran lugares comunes sin llegar a solución operativa alguna.

Resulta particularmente importante, asimismo, la confrontación de proyectos gubernamentales con proyectos populares surgidos de la experiencia cotidiana. Una acción de este tipo representaría un vuelco formidable respecto a la tradicional actitud paternalista del Estado, haciendo aflorar el inagotable repertorio de soluciones que visualiza quien está presionado por necesidades vitales.

La participación popular debe ser lograda bajo las condiciones en que puede ser más efectiva. Se requiere de un permanente flujo de información de ida y vuelta, de aportar elementos que permitan pasar de la simple petición o reivindicación a un auténtico diálogo sobre problemas contingentes y de estrategia a futuro.

Esto es fácil de decir, peró extremadamente complejo de realizar pues a los planificadores les resulta difícil imaginar que existan otras formas de visualizar la realidad y muchas veces subestiman todo aquello que no viene respaldado por conceptos teóricos sofisticados. 
4.3 Integración de los sistemas de gestión y administración metropolitana

Las acciones sugeridas en los puntos anteriores difícilmente podrían ponerse en práctica si no se cuenta con una estructura política y administrativa adecuad a para ello. Además de la problemática estructural que afecta эl desarrollo metropolitano de la ciudad de México, mucho se ha argumentado sobre la ineficiencia de los aparatos del Estado para actuar coherentemente en este espacio. Múltiples dependencias, organismos y empresas estatales del gobierno federal, del Departamento del Distrito Federal, leí gobierno del estado de México y de los municipios metropolitanos, ictúan en la planeación territorial y en la provisión y administración de los servicios públicos y equipamiento sin que haya sido posible, no se diga sontrolar, sino sólo contar con una visión del todo metropolitano. Las consecuencias de esta falta de coordinación y coherencia son múltiples. Cada sntidad plan ifica su porción de territorio autónomamente; existen múltiles sistemas de transporte, unos públicos y otros privados, concesionaios o tolerados, que actúan descoordinadamente; los sistemas fiscales y arifarios no se corresponden y tienen objetivos y criterios de aplicación iistintos; los sistemas de educación, salud, seguridad pública, recreación, stc., responden a prioridades que poco tienen que ver con un esquema ntegral de crecimiento metropolitano. En suma, los sistemas jurídicos, ıdministrativos y financieros, y los criterios políticos, económicos, sociales $\gamma$ técnicos con que se administra y gestiona el AMCM, no han sido elaboados para alcanzar metas de desarrollo metropolitano.

Resulta pues fundamental llevar a cabo una estrategia de reordenaniento político-administrativo con el objeto de establecer bases firmes para ina planeación, administración y gestión más coherente del espacio meropolitano. Para dicho efecto, pensamos que se requiere crear un orgadismo metropolitano que sea capaz de integrar y sintetizar las múltiples niciativas provenientes de los niveles de gobierno y de la sociedad como m todo.

Las funciones generales de dicho órgano deberían ser: planificar, cooriinar y poner en marcha las acciones definidas como de interés metropoitano. Es decir, aquellas acciones que por sus propias características no jueden quedar sujetas al interés de un sólo municipio, sino del conglonerado metropolitano.

En espera de estudios que permitan determinar los criterios para la dentificación de los servicios y actividades de interés metropolitano, paece adecuado considerar como de este tipo la planeación integral de su iesarrollo; el control de los usos del suelo; el sistema integrado de transjorte público; los servicios públicos de agua potable, drenaje, alcantarilado y gas, recolección y tratamiento de basura; el mejoramiento ambiental t los recursos hídricos, y la seguridad pública.

Otros servicios y actividades como la educación básica, la salud, la 
asistencia social y la recreación y el turismo requerirían de un análisis más profundo para determinar si deben ser considerados parcial o totalmente de nivel metropolitano.

La organización ejecutiva del órgano metropolitano debería basarse en una estructura lo más democrática posible, conformada por los representantes políticos de la población a nivel municipal y por los organismos comunitarios (partidos políticos, sociedades gremiales, asociaciones de pobladores, cámaras sectoriales, etc.) que representan los diversos intereses de la sociedad civil. Asimismo, por representantes de los gobiernos estatales y federal que, desde una perspectiva básicamente sectorial, inciden en el desarrollo metropolitano. En este sentido, además de las funciones sugeridas, seguramente absorbería las asignadas a los Comités de Planeación del Desarrollo (Coplades).

La representatividad en el consejo, comité o asamblea metropolitana, y la democratización de sus decisiones, son probablemente los ejes centrales de su acción institucional.

El autoritarismo y la verticalidad en las decisiones han mostrado su absoluta incapacidad para dar respuesta a los múltiples problemas que aquejan nuestra metrópolis.

La gestión local del espacio metropolitano ha dejado de ser ideal, para convertirse en la vía más idónea para reducir las desigualdades y la injusticia en la distribución de los bienes y servicios metropolitanos.

Debemos aclarar que la creación de un ente metropolitano de gestión socioespacial no basta para resolver los problemas. Se requiere asimismo que, a través de éste, y a través de una redefinición real del municipio como nivel de gobierno con máxima autoridad y recursos en cuestiones locales, se restructuren los esquemas jurídicos, administrativos, financieros y sobre todo políticos de relación entre niveles de gobierno superiores.

Es decir, una mayor y más decidida intervención estatal en la problemática metropolitana, con un enfoque de servicio público y justicia social por encima del carácter impositivo y empresarial actual, y con una clara apertura a la gestión comunitaria del espacio, más que un problema de técnica jurídico-administrativa, es un problema político.

Un ente de esta naturaleza, con las funciones y capacidades ejecutivas sugeridas, difícilmente surgirá por iniciativa propia del Estado. Será producto fundamentalmente de una lucha política por democratizar y dar coherencia a la gestión metropolitana.

Dentro de este proceso, el papel más importante corresponde a los partidos políticos y a todas aquellas organizaciones más directamente involucradas en la problemática espacial metropolitana.

La creación de un ente metropolitano, con una base jurídica, administrativa y financiera adecuadas al cumplimiento de sus funciones, representa sólo uno de los elementos necesarios para que se logre avanzar desde el Estado en las transformaciones socioespaciales. Se requiere, asi- 
mismo, impulsar políticamente cambios en las concepciones actuales de la administración municipal y estatal.

Destaca en este sentido el carácter de servicio público y justicia social que debería privar en los organismos y empresas estatales vinculados a los servicios metropolitanos, por encima de su actual esquema tecnocrático y eficientista.

La miseria urbana existe como producto de una estructura de explotación y segregación, en la que participa también el Estado, al aplicar sus planes, inversiones, sistemas fiscales y tarifarios bajo la lógica del mercado. El discurso de la eficiencia, la técnica y el saneamiento financiero de los organismos y empresas estatales adquiere un carácter ideológico, cuyas consecuencias son la ampliación de la brecha entre la ciudad moderna, rica, y su periferia pobre.

La prestación de servicios y bienes de consumo colectivo, la fiscalización y la definición de tarifas, deberían contemplar la eficiencia técnica y financiera al nivel del conjunto de acciones en la metrópolis y no al de cada empresa u organismo. El objetivo sería eliminar el concepto de que sólo viva en la metrópolis aquél que pueda pagar sus costos, cambiándolo por el de los que más tienen y que son siempre los beneficiados por la urbanización, transfieran recursos - por la vía del Estado- a los que tienen menos y que casi nunca son beneficiados por las obras de urbanización.

Dicho organismo metropolitano no pretendería convertirse en sustituto de los poderes establecidos constitucionalmente en el AMCM. Sin embargo, se requeriría de la transformación del actual régimen de gobierno en el Distrito Federal y de tal manera que su carácter de organismo de planeación y administración de nivel local no fuera trastocado por la inexistencia de los niveles de gobierno estatal y municipal en el Distrito Federal.

En este sentido, lo que resulta importante es que el AMCM como unidad geoeconómica tenga un régimen de gobierno estatal y municipal.

El equilibrio político entre entidades federativas y el proceso de expansión de la metrópolis previsto para el futuro, nos hacen pensar que no es clara la conveniencia de unificar toda el AMCM en un solo estado; explorar la posibilidad de hacer del Distrito Federal una nueva entidad federativa, reduciendo el espacio del Distrito Federal a un área bastante más reducida o trasladándolo a otra localización, haría que este nuevo estado compartiera el espacio metropolitano con el estado de México y en el futuro, incluiría probablemente otras entidades vecinas.

Todas las acciones sugeridas apuntan a una restructuración profunda del AMCM, hacia afuera, en su contexto nacional y regional, así como en su conformación interna.

De antemano sabemos que en México no ha existido "clima político" para dar respuestas realmente comprometidas a un problema que ha ido expandiéndose hasta afectar muy severamente el desarrollo del país. 
No obstante, pensamos que se han ido acumulando las condiciones (por la vía de la agudización de los problemas), que nos conducen a la posibilidad de que se tomen grandes decisiones que enfrenten, aunque sea parcialmente, la estructura misma de los problemas.

En todo caso, lo que está claro es que la política urbana de paliativos y parches ha perdido su eficacia política, lo que implica que existe un alto riesgo de que se revierta en contra de la estabilidad social de la mayor metrópolis del país y del mundo.

\section{Bibliografía}

Bernardés, L, (1976), “Áreas metropolitanas e seu Planejamento" en Vacowicz, M. (org.), Desenvolvimento e Política Urbana IBAM, Río de Janeiro.

Brasileiro, A. (1976), "Regiao Metropolitana do Grande Rio", Servicos de Interesse Comun. IPEA, Brasilia.

Caffe, A. (1981), Planejamento Metropolitano e Autonomía Municipal no Direito Brasileiro, Jose Buskatsky, Sao Paulo.

Castells, M. (1978), La cuestión urbana, Siglo XXI, México.

Fidel, C. A. Iracheta y E. Soms (1982), "Límites internos y externos de la planeación urbana" en Memoria de la reunión nacional sobre la problemática del suelo de México, Tlaxcala.

Garza, G. y M. Schteingart (1978), "Mexico City: The Emerging Megalopolis", en Cornelius W. y Kemper R. (eds.), Metropolitan Latin America. The Challenge and the Response, Latin American Urban Research, vol. 6, SAGE Publications, Londres.

Geisse, G. y J.L. Coraggio (1972), "Áreas metropolitanas y desarrollo nacional" en Geisse G. y J. Hardoy, (comps.). Políticas de desarrollo urbano y regional en América Latina, Ediciones SIAP, Buenos Aires.

Harvey, D. (1973), Social Justice and the City, Routledge and Kegan Paul, Londres. Iracheta, A. (1982), "El problema del transporte en las áreas metropolitanas del estado de México", UAEM Toluca. (1984), El suelo, recurso estratégico para el desarrollo urbano, UAEM, Toluca.

(1987), "Algunas consideraciones sobre el problema del suelo para vivienda en la ZMCM", EPUR, UAEM, México.

(1988), Hacia una planeación urbana crítica, UAM-ED, Gernika, México (en prensa).

Soms, E., La hiperurbanización en el valle de México, UAM-A. México. 